\title{
Performance and Stability of Different Monoecious Hemp Cultivars in a Multi-Environments Trial in North-Eastern Italy
}

\author{
Claudio Ferfuia ${ }^{1, *}$, Fabio Zuliani ${ }^{1}$, Francesco Danuso ${ }^{1}$, Barbara Piani ${ }^{1}$, Costantino Cattivello ${ }^{2}$, Gaia Dorigo ${ }^{2}$ \\ and Mario Baldini ${ }^{1}$ (D)
}

1 DI4A, Department of Agricultural, Food, Environmental and Animal Sciences, University of Udine, Via delle Scienze, 206, 33100 Udine, Italy; fabio.zuliani@uniud.it (F.Z.); francesco.danuso@uniud.it (F.D.); barbara.piani@uniud.it (B.P.); mario.baldini@uniud.it (M.B.)

2 ERSA FVG, Servizio Fitosanitarioe Chimico, Ricerca, Sperimentazione ed Assistenza Tecnica, Via Sabbatini, 5, Pozzuolo del Friuli, 33050 Udine, Italy; costantino.cattivello@ersa.fvg.it (C.C.); gaia.dorigo@ersa.fvg.it (G.D.)

* Correspondence: claudio.ferfuia@uniud.it

Citation: Ferfuia, C.; Zuliani, F.; Danuso, F.; Piani, B.; Cattivello, C.; Dorigo, G.; Baldini, M. Performance and Stability of Different Monoecious Hemp Cultivars in a Multi-Environments Trial in North-Eastern Italy. Agronomy 2021, 11, 1424. https://doi.org/ 10.3390/agronomy11071424

Academic Editor: Nikos Tzortzakis

Received: 21 June 2021

Accepted: 14 July 2021

Published: 16 July 2021

Publisher's Note: MDPI stays neutral with regard to jurisdictional claims in published maps and institutional affiliations.

Copyright: (c) 2021 by the authors. Licensee MDPI, Basel, Switzerland. This article is an open access article distributed under the terms and conditions of the Creative Commons Attribution (CC BY) license (https:/ / creativecommons.org/licenses/by/ $4.0 /)$.

\begin{abstract}
The seed yield in hemp (Cannabis sativa L.) is strongly influenced, besides by genotype, by environment and the genotype $x$ environment interaction, so establishing the fitness and stability of hemp cultivars in multiple environments is necessary. The purpose of this study was to investigate the performance and degree of stability and variance of seed yield, the main related traits, and the correlation among the traits in five hemp monoecious cultivars cultivated in six different environments. The environments resulted from the combination of four locations, two years, and two delayed sowings in a Mediterranean area of north-eastern Italy, and the stability index of the weighted average of absolute scores (WAAS) was used in order to identify the most productive and stable genotypes on the basis of their deviation from the average performance across environments. In this studied area, early varieties, such as Fedora and Felina, proved to be the best performing and stable for seed yield and both increased their yield in correspondence to delayed sowing times, opening up the possibility of cultivating hemp as a second crop. Among the climate parameters, high temperatures during the early grain filling period led to a progressive decrease in seed yield. For a dual-purpose crop, a good compromise could be a late monoecious cultivar (like Futura, in the present experiment), which, if sown early, could certainly provide notable biomass production and acceptable seed yield.
\end{abstract}

Keywords: multi-environment trials (MET); genotype x environment interaction (GEI); stability index; weighted average of absolute scores (WAAS); Cannabis sativa L.

\section{Introduction}

Industrial hemp (Cannabis sativa L.) is considered to be a multi-purpose crop, with many current and potential end uses as food, energy, textile, paper, biocomposite, medical usage, fodder, and many other industrial products [1,2], as well as being used as a pollutantremoving and marginal land restoration crop [3-8]. The total hemp area in Europe reached more than 50,000 ha in 2018, with a forecast for further growth, and an increase of as much as $70 \%$ compared to the average of the last 5 years, and of $3.3 \%$ compared to 2017. Among EU countries, France is the country with by far the largest hemp area (37\%), followed by Italy, with $8 \%$, equivalent to about 4000 ha [9]. The varieties of hemp permitted for cultivation in the EU are registered in the common catalogue of varieties of agricultural plant species, which, as per regulation (EU) 1307/2013 [10], must have a level of tetrahydrocannabinol (THC) not exceeding $2 \%$, and of which there are currently just over seventy (EU Plant Variety Database) [11]. The main product of hemp in the recent past was the production of short fibre and dusted and calibrated shives for industrial application, while interest in the production of seeds for food purposes has significantly increased recently, as evidenced by the about 20,300 tons of hemp seed produced in the EU in 2018, of 
which $90 \%$ was processed within the European borders. In Italy, interest in the production of hemp seed as food started after the 2009 Circular of the Ministry of Health [12], which recognises the lawfulness of the alimentary use of hemp seed and derivatives, recently improved the definition of the maximum levels of tetrahydrocannabinol (THC) allowed in hemp food [13]. Dioecious hemp varieties are decidedly unsuitable for seed production due to their lack of uniformity, excessive size, and the consequent difficulties in mechanical harvesting and the natural presence of about $50 \%$ of male plants, which are useless and as harmful as weeds for the remaining female plants. For this reason, the monoecious varieties selected specifically for seed production and that are suitable for specific environments are required. It is well known that in hemp, the seed yield trait is strongly influenced, besides by genotype, by environment and by the genotype $x$ environment interaction, the effect of which often depends on the origin and breeding environment where the specific cultivar was obtained. Out of the more than 70 varieties present in the EU register, about 10 are of Italian origin, of which very few are monoecious and are mainly selected and used for fibre production and/or for medical cosmetic use [14]. Italy has a great tradition in the selection and cultivation of dioecious varieties for fibre production [15-20], while much less has been investigated on the potential of monoecious varieties for seed yield, except for a few evaluations of dual-purpose use (seed and fibre) [21-25]. No contribution has had the aim of establishing the fitness and suitability of the current monoecious cultivars for seed yield in multiple Italian environments in order to assess the degree of stability and variance of seed yield and the related traits in the phenotypes of a cultivar.

As is known, the phenotype depends on the cumulative effect of the genotype (G), the environment (E) and their interaction (GEI). While the effects of genotype are quite constant (cumulative actions of many genes), the environment, which varies over locations and years, is the major factor that affects phenotypic behaviour. The relative performance of a given genotype (e.g., grain yield or biomass yield) could vary across environments, and these differences are attributed to GEI, in addition to the simple effects of the E. The genotypes that respond less to altering environmental conditions are stable and have the probability of achieving a similar performance over a range of environments. Thus, GEI plays a key role in crop improvement. Several statistical methods had been developed, starting with Finlay and Wilkinson's [26] regression model, to assess the stability of genotypes. Nowadays, the most commonly used models to describe genotype stability are the additive main effects and multiplicative interaction (AMMI) model [27] and the genotype main effects and genotype $x$ environment interaction effects (GGE) model. More recently, Olivoto et al. [28] proposed a new stability index called the WAAS (weighted average of absolute scores), in which the most stable genotypes deviate the least from the average performance across environments. With WAAS, it is also possible to evaluate the environmental performance from the point of view of discrimination abilities and productivity. One of the most important advantages of WAAS, with respect to the other methods, is that it can be used for a joint interpretation of stability and productivity.

Many environmental factors could be responsible for the inconstancy in hemp seed yield, as previously reported by Tang et al. [22], and, among these, differences in temperature during the grain filling period could affect the formation, filling, seed yield, and quality, as already reported about hemp [25,29] and other crops, such as rice [30], maize [31], soybean [32], and sunflower [33,34]. A review of the recent literature shows that very few attempts have been made to study the GEI effect on grain and total biomass yield using multivariate statistical methods in hemp (e.g., Campbell et al. [35]).

The main purpose of this study was to investigate the production potential, performance and stability of different hemp monoecious genotypes cultivated across several environments and years in a Mediterranean area of north-eastern Italy using the WAAS index. The WAAS index allows for the stable hemp genotypes for the yield to be identified and for the categorisation of the similarly responding genotypes and environment. This approach allows for more detailed information to be gained on cultivar suitability for 
seed yield in northern Italy and for general information about hemp genotypes and crop management techniques to be obtained for the specific environment.

\section{Materials and Methods}

\subsection{Plant Materials}

Five monoecious cultivars, namely Fedora, Felina, Ferimon, Futura, and Zenit, were evaluated under six different environments. The main characteristics of these monoecious genotypes are reported in Table 1 . These cultivars have a different cycle duration under these environmental conditions, are listed in the EU database of registered hemp varieties, and have been used in several Italian field trials $[17,20,22]$.

Table 1. Main characteristics of the five monoecious cultivars tested.

\begin{tabular}{cccc}
\hline Cultivar & Origin & Maturity Group & Main Use \\
\hline Fedora 17 & France & Early & Seed and biomass \\
Felina 32 & France & Medium-early & Seed and biomass \\
Ferimon & France & Medium-early & Seed and biomass \\
Futura 75 & France & Late & Seed and biomass \\
Zenit & Romania & Early & Seed \\
\hline
\end{tabular}

\subsection{Environments Characteristics}

The six studied environments resulted from the combination of four locations (S. Osvaldo (SO); Verzegnis (VE); Campoformido (CF); Majano (MA)) and two years (2018 and 2019). Two delayed seasonal sowings (D) were also carried out in SO and VE in 2019. In particular, the six environments considered were the following: SO2019, VE2019, CF2018, MA2018, SO2019D, and VE2019D.

Details of the main meteorological parameters recorded during the hemp crop cycle for each location are reported below and represented in Figure 1.

S. Osvaldo (SO) - The trial was conducted at the experimental farm of Udine University in S. Osvaldo, UD (N 46 $04^{\prime}, \mathrm{E} 13^{\circ} 23^{\prime}, 109 \mathrm{~m}$ a.s.l.), located on the medium plains of Friuli Venezia Giulia region. The site is characterised by a shallow soil (about $50 \mathrm{~cm}$ ) with a sandy silt texture (average sand, silt, and clay are 40,43 , and $17 \%$, respectively), with $22 \%$ gravel and a $\mathrm{pH}$ of 7.3. Meteorological data was collected by the weather station of Udine Sud, very close to the site of the experimental trials. On average, rainfall during the crop cycle $(610 \mathrm{~mm})$ was lower than the average of the last 10 years, concentrated in May $(302 \mathrm{~mm})$ and in the first 10 days of September $(136 \mathrm{~mm})$. Concerning average maximum temperatures, the hottest periods, with peaks of $32{ }^{\circ} \mathrm{C}$, were in the last 10 days of June, the last 10 days of July, and the last 10 days of August. The lowest minimum temperatures $\left(9-10^{\circ} \mathrm{C}\right)$ were recorded in the first 10 days of May and in the first and last 10 days of October.

Verzegnis (VE) - The trial was conducted in Verzegnis municipality, UD ( $\mathrm{N} 46^{\circ} 38^{\prime}, \mathrm{E}$ $13^{\circ} 01^{\prime}, 320 \mathrm{~m}$ a.s.l.) located on the foothills of Friuli Venezia Giulia. The site has a very shallow soil (about $35 \mathrm{~cm}$ ) with a silty loam texture (average sand, silt, and clay are 31, 62 and $7 \%$, respectively), with $17 \%$ gravel and a $\mathrm{pH}$ of 8.0 . The locality has a continental temperate climate. Verzegnis meteorological was data obtained by the weather station of Tolmezzo, about $2.5 \mathrm{~km}$ far from the experimental trials. On average, rainfall during the crop cycle $(618 \mathrm{~mm})$ was lower than the average of the last 10 years, concentrated in May $(216 \mathrm{~mm})$ and October $(140 \mathrm{~mm})$. The maximum temperatures recorded reached an average of $32{ }^{\circ} \mathrm{C}$ in the second and third 10 days of June and in the last 10 days of July and August, remaining below $30^{\circ} \mathrm{C}$ during the rest of the summer season. Minimum values $\left(7-8{ }^{\circ} \mathrm{C}\right)$ were reached in the first 10 days of May and October. 
Campoformido (CF) - The trial was conducted in Campoformido municipality, UD (N $46^{\circ} 01^{\prime}, \mathrm{E} 13^{\circ} 08^{\prime}, 79 \mathrm{~m}$ a.s.l.), located on the medium plains of Friuli Venezia Giulia region. The site has a shallow soil layer (about $0.6 \mathrm{~m}$ ) with a loamy texture (average sand, silt, and clay are 33,46 , and $21 \%$, respectively) and a $\mathrm{pH}$ of 7.8 . The location has a continental temperate climate, with average annual minimum, average, and maximum temperatures of $12.2,13.5$, and $14.7^{\circ} \mathrm{C}$, respectively (referring to the period of 1990-2018). Meteorological data was obtained by the weather station of Colloredo di Prato (UD), about $3.5 \mathrm{~km}$ far from the experimental trials. The average annual rainfall is $1466 \mathrm{~mm}$ (referring to the period 1990-2018). Spring had rainfall in line with the long-term averages, with the exception of the mid-June period, which was dry. Summer was rainy on average, with the exception of the first 10 days of August and the second half of September. There was abundant rainfall in the first and third 10 days of October, with concentrated, rather intense showers.
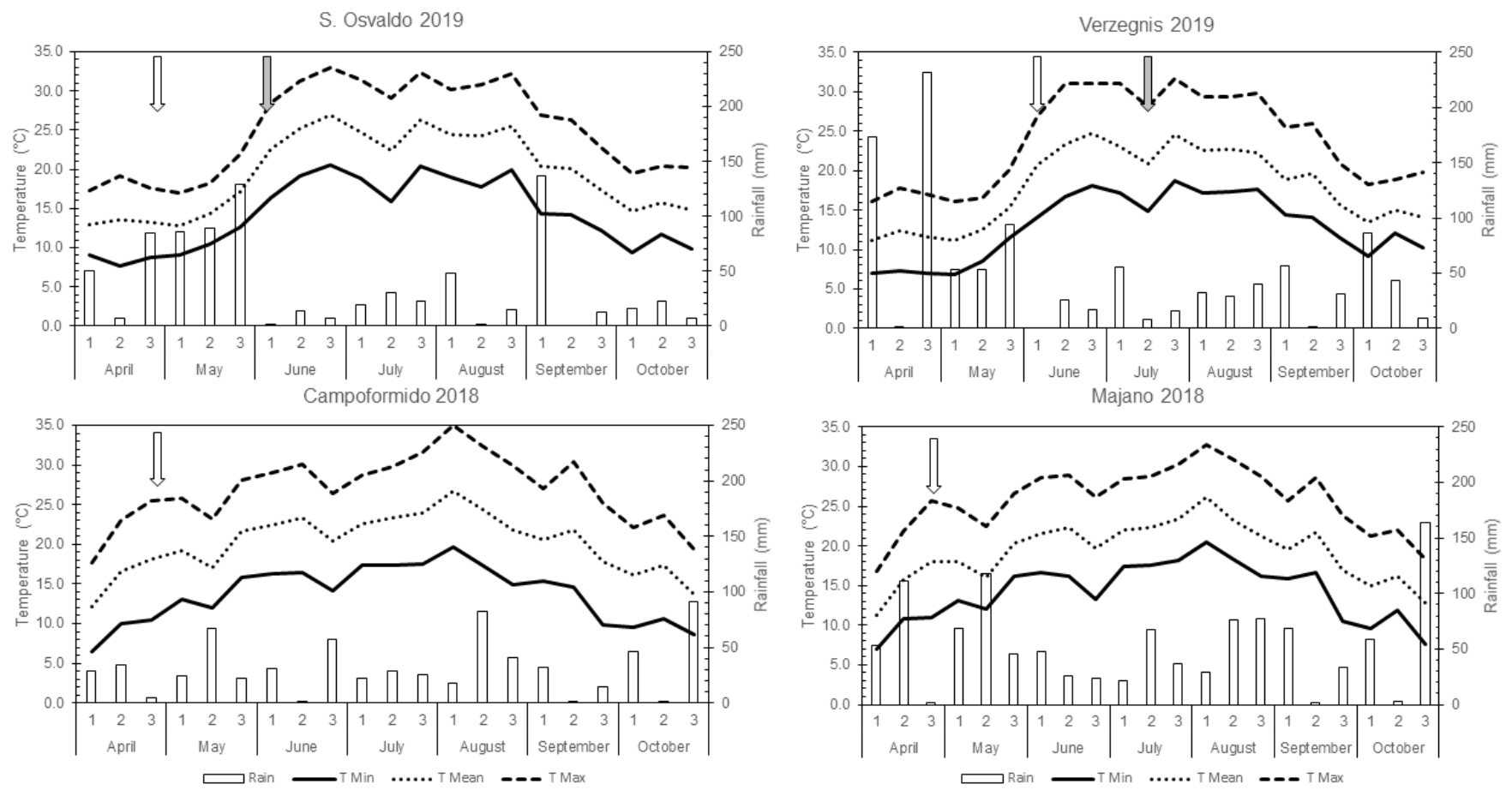

Figure 1. Daily mean temperature (T Mean), mean maximum temperature (T Max), minimum temperature (T Min), and total rainfall during the growing seasons (10-day values). White arrows represent conventional sowing time. Grey arrows represent delayed sowing time in SO2019D and VE2019D.

Majano (MA)-The trial was conducted in Majano, Gemona, UD (N 46 $12^{\prime}$, E $13^{\circ} 03^{\prime}$, $160 \mathrm{~m}$ a.s.l.) located on the high plains of Friuli Venezia Giulia region. The site has a deep soil level (more than $150 \mathrm{~cm}$ ) with a silty loam texture (average sand, silt, and clay are 30, 61 , and $9 \%$, respectively), a shallow water table, and a $\mathrm{pH}$ of 7.9. Meteorological data was obtained by the weather station of Rivoli di Osoppo, $2.6 \mathrm{~km}$ far from the experimental trials. The location has a continental temperate climate, with average annual minimum, average, and maximum temperatures of $8.5,12.5$, and $18.7^{\circ} \mathrm{C}$, respectively (referring to the period of 1999-2018). The average annual rainfall is $2123 \mathrm{~mm}$ (referring to the period of 2005-2018). The months involved in the trial were on average wetter than in Campoformido, with the exception of the last 10 days of July, early August, and the second 10 days of September and October, which were periods with low rainfall. Very abundant rainfall concentrated in a short amount of time was recorded at the end of October. 
Details of the main physical-chemical characteristics of soil for the above locations are reported in the Supplemental Materials (Table S1). Soil analysis was carried out by the Agricultural Chemistry, Pesticides, and Contaminants Laboratory (Laboratorio di chimica agraria, fitofarmaci e contaminanti) of Friuli Venezia Giulia Regional Agricultural Services (Agenzia regionale per lo Sviluppo Rurale del Friuli Venezia Giulia-ERSA), Udine (Italy). Monthly main climatic parameters recorded during the hemp crop cycle for each location of the previous 28-year period (1992-2019) are reported in the Supplemental Materials (Table S2).

A plot seeder was used for sowing at a depth of about $3 \mathrm{~cm}$, in rows $0.15 \mathrm{~m}$ apart, using approximately 120 viable seeds per $\mathrm{m}^{2}$. The total fertilization, ranging from 80 to $100 \mathrm{~kg} \mathrm{ha}^{-1}$ of $\mathrm{N}$, was applied as urea, with the exception of the CF location, where only mature manure was applied as organic fertilization. The conventional sowing time for all locations was at the end of April, the management approach commonly adopted in the region for hemp cultivation. In addition to the above conventional sowing, a delayed sowing was done in June at the SO2019 and VE2019 environments, identified as SO2019D and VE2019D. In each environment, weed control was not necessary, with the exception of very limited areas, where manual weeding was performed. All the trials were conducted with a deficit irrigation supplement with sprinkler irrigations, with the exception of Majano, where the presence of a superficial water table, reachable by the hemp roots even in the summer period, made irrigation unnecessary. The irrigation volume was $30 \mathrm{~mm}$, which is the usual amount adopted by farmers in this specific crop and pedo-climatic conditions. Further details on the management techniques are reported in the Supplemental Materials (Table S3).

\subsection{Data Collection and Trait Determination}

The emergence date for each genotype was set when about $50 \%$ of the expected plants showed the hypocotyl. Emergence was sufficiently uniform for all cultivars in all environments, from about 5 to 9 days after sowing. A total of 10 representative plants in the same area were randomly chosen in order to check, once a week, the phenological stage, to determine the full flowering (FF) and seed maturity stages (SM), as in Mediavilla et al. [36]. Harvesting, performed about ten days after the seed maturity stage for each cultivar which corresponded to 50\% of hard seed, as in Mediavilla et al. [36], started in August with the earliest varieties and ended in late October in all environments. All plants in a $2.0 \mathrm{~m}^{2}$ area of each experimental unit were cut manually at $10 \mathrm{~cm}$ from the soil surface. Plant height was measured on 10 randomly chosen plants, from the base to the top of the inflorescence, and all harvested plants were divided into two fractions: stems and inflorescence, weighed separately. These were oven-dried at $70{ }^{\circ} \mathrm{C}$ until a constant weight was reached in order to evaluate the aboveground and stem total dry biomass. The seeds, separated from the inflorescence by means of a threshing machine, were cleaned, and their weight, specific weight, and yield were determined. A representative sub-sample of the dried seed of each cultivar and environment was stored at $4{ }^{\circ} \mathrm{C}$ for further chemical analysis. Briefly, the hemp seeds were ground in a coffee mill for $30 \mathrm{~s}$, and after grinding, ether extracts were obtained by treating the hemp seed for $12 \mathrm{~h}$ with petroleum ether (b.p. $40-60^{\circ} \mathrm{C}$ ) using a Soxhlet apparatus. The petroleum ether was then removed by evaporation under a nitrogen gas flow, and the oil was dried over anhydrous sodium sulphate $\left(\mathrm{Na}_{2} \mathrm{SO}_{4}\right)$, filtered, and quantified. Each sample was extracted in triplicate. Seed and plant analysis was carried out by the Analytical Chemistry Laboratory, Department of Agricultural, Food, Environmental and Animal Sciences (DI4A) of Udine University, Udine (Italy). The accumulation of the growing degree days (GDD), above a base temperature of $10{ }^{\circ} \mathrm{C}(\mathrm{Tb}$ [29]), was calculated using the following formula:

$$
\mathrm{GDD}=\Sigma[(\operatorname{Tmax}+\mathrm{Tmin}) / 2]-\mathrm{Tb}
$$

where Tmax and Tmin are the daily maximum and minimum temperatures, respectively, in ${ }^{\circ} \mathrm{C}$. 
In summary, the five hemp monoecious cultivars were tested over six environments (4 different locations and 2 years) in north-eastern Italy. Eight traits were measured under these conditions, namely hypocotyl emergence-full flowering and hypocotyl emergenceseed maturity periods, seed yield, seed unit weight, specific seed weight, seed oil content, plant height, and total aboveground biomass

\subsection{Experimental Design and Statistical Analysis}

In each location, the trial was sown in a randomised block with four replications, where the experimental factor was the cultivar as the fixed effect, with each experimental unit being about $35 \mathrm{~m}^{2}$. Statistical analysis was conducted using the software $\mathrm{R}$ (version 4.0.2 [37]) with the metan (multi-environment trials analysis, version 1.7.0) package, developed by Olivoto and Lúcio [38]. The Shapiro-Wilk normality test was performed to test the normality condition. Primarily, individual analysis of variance (ANOVA) for each environment was performed to check the genotype effect in each environment, followed by an analysis of joint variance to check the GEI. If the GEI is significant, then it is reasonable to proceed with some stability analysis to explore the interaction and then the weighted average of absolute cores (WAAS). The WAAS is a stability analysis proposed by Olivoto et al. [28], and it derives from the single value decomposition of the matrix of best linear unbiased predictions for the GEI effects generated by a linear mixed-effect model. The cultivar that deviates the least from the average performance across environments, and with the lowest WAAS value, is considered the most stable. Pearson correlation coefficients were also computed to measure the strength of linear association among investigated traits.

\section{Results}

The mean seed yield varied significantly, from 0.19 to $1.15 \mathrm{Mg} \mathrm{ha}^{-1}$, and these observed extreme values were mainly contributed to by the environment (Table 2). Environment effects, in fact, explained $82 \%$ of character variance, with $12 \%$ of variance attributed to the GEI effects and a low but significant genetic effect (4\%) (Table 3$)$. The individual analysis (in each environment) revealed that all environments showed significant differences for genotype effects (data not shown). The seed yield obtained at VE2019D (1.09 $\mathrm{Mg} \mathrm{ha}^{-1}$ ) and SO2019D (1.15 $\left.\mathrm{Mg} \mathrm{ha}^{-1}\right)$, with a delayed sowing time, resulted in the highest of the trial, with an increase of 55 and 17\%, respectively, with respect to the performance obtained with the conventional sowing time in the same locations. However, not all cultivars were affected equally by the delayed sowing time, as can be seen in the seed yield reaction (Figure 2), in which a particularly divergent GEI for seed yield is evident, which is in agreement with other authors [35,39]. In particular, Ferimon, Fedora, and Felina showed the largest positive response to the delayed sowing time. The responses of Zenit and Futura were completely different, significantly decreasing their rank for seed yield in correspondence to delayed sowing times.

Table 2. Overall mean (OV), min, and max values among environments (ENV) and genotypes (GEN) for the eight studied traits.

\begin{tabular}{|c|c|c|c|c|c|c|c|c|}
\hline Parameter & $\mathrm{SY}^{\mathrm{a}}\left(\mathrm{Mg} \mathrm{ha}^{-1}\right)$ & $\operatorname{SOC}^{b}(\%)$ & $\operatorname{ABY}^{\mathrm{c}}\left(\mathrm{Mg} \mathrm{ha}^{-1}\right)$ & $\mathrm{PH}^{\mathrm{d}}(\mathrm{cm})$ & $1000-\mathrm{SW}^{\mathrm{e}}(\mathrm{g})$ & $\mathrm{HL} \mathrm{SW}^{\mathrm{f}}\left(\mathrm{kg} \mathrm{hL}^{-1}\right)$ & $\mathrm{EF}^{\mathrm{g}}$ (days) & EPM $^{\text {h }}$ (days) \\
\hline $\mathrm{OV}$ & 0.78 & 18.87 & 3.92 & 157 & 8.86 & 0.32 & 61 & 102 \\
\hline MinENV & CF2018 (0.19) & CF2018 (6.71) & SO2019D (2.08) & VE2019 (115) & MA2018 (5.82) & CF2018 (0.23) & VE2019D (44) & VE2019D (84) \\
\hline MaxENV & SO2019D (1.15) & VE2019D (28.19) & MA2018 (6.97) & MA2018 (201) & VE2019D (12.31) & VE2019D (0.41) & MA2018 (72) & MA2018 (128.4) \\
\hline MinGEN & Zenit $(0.50)$ & Fedora (15.64) & Zenit (2.88) & Ferimon (140) & Felina (6.19) & Felina $(0.27)$ & Fedora (53) & Fedora (94) \\
\hline MaxGEN & Fedora (0.98) & Futura (25.19) & Futura (4.99) & Futura (183) & Zenit (12.13) & Futura (0.41) & Futura (75) & Futura (112) \\
\hline
\end{tabular}

${ }^{\text {a }}$ Seed yield; ${ }^{b}$ seed oil content; ${ }^{c}$ aboveground biomass yield; ${ }^{d}$ plant height; ${ }^{\mathrm{e}}$ seed weight; ${ }^{\mathrm{f}}$ hectolitre seed weight; ${ }^{\mathrm{g}}$ duration from emergence to flowering period; ${ }^{\mathrm{h}}$ duration from emergence to physiological maturity period. 
Table 3. Joint ANOVA ( $p$-value) and variance explained (\%) by environment (ENV), genotype (GEN) and GEI for the eight studied traits.

\begin{tabular}{|c|c|c|c|c|c|c|c|c|c|c|c|c|c|c|c|c|c|}
\hline \multirow[t]{2}{*}{$\begin{array}{l}\text { Source of } \\
\text { Variation }\end{array}$} & \multirow[t]{2}{*}{$D f^{a}$} & \multicolumn{2}{|c|}{$S Y^{b}$} & \multicolumn{2}{|c|}{$\operatorname{SOC}^{\mathrm{c}}$} & \multicolumn{2}{|c|}{$A B Y^{d}$} & \multicolumn{2}{|c|}{$\mathrm{PH}^{\mathrm{e}}$} & \multicolumn{2}{|c|}{$1000-S W^{f}$} & \multicolumn{2}{|c|}{ HL SW $\mathrm{g}$} & \multicolumn{2}{|c|}{$\mathrm{EF}^{\mathrm{h}}$} & \multicolumn{2}{|c|}{$\operatorname{EPM}^{\mathrm{i}}$} \\
\hline & & $p$ & $\%^{1}$ & $p$ & $\%^{1}$ & $p$ & $\%^{1}$ & $p$ & $\%^{1}$ & $p$ & $\%^{1}$ & $p$ & $\%^{1}$ & $p$ & $\% 1$ & $p$ & $\%^{1}$ \\
\hline ENV & 5 & $* * *$ & 82 & $* * *$ & 77 & $* * *$ & 60 & $* * *$ & 47 & $* * *$ & 38 & $* * *$ & 78 & $* * *$ & 27 & $* * *$ & 59 \\
\hline GEN & 4 & $* * *$ & 4 & $* * *$ & 7 & $* * *$ & 15 & $* * *$ & 40 & $* * *$ & 24 & $* * *$ & 3 & $* * *$ & 61 & $* * *$ & 30 \\
\hline GEI & 20 & $* * *$ & 12 & $* * *$ & 13 & $* * *$ & 20 & $* * *$ & 12 & $* * *$ & 25 & $* * *$ & 9 & $* * *$ & 12 & $* * *$ & 11 \\
\hline
\end{tabular}

${ }^{a}$ Degree of freedom; ${ }^{b}$ seed yield; ${ }^{c}$ seed oil content; ${ }^{d}$ aboveground biomass yield; ${ }^{\mathrm{e}}$ plant height; ${ }^{\mathrm{f}}$ seed weight; ${ }^{\mathrm{g}}$ hectolitre seed weight; $\mathrm{h}$ duration from emergence to flowering period; ${ }^{\mathrm{i}}$ duration from emergence to physiological maturity period. ${ }^{1} \%=$ Percent variance explained; ${ }^{* * *}$ significant at $p \leq 0.001$.

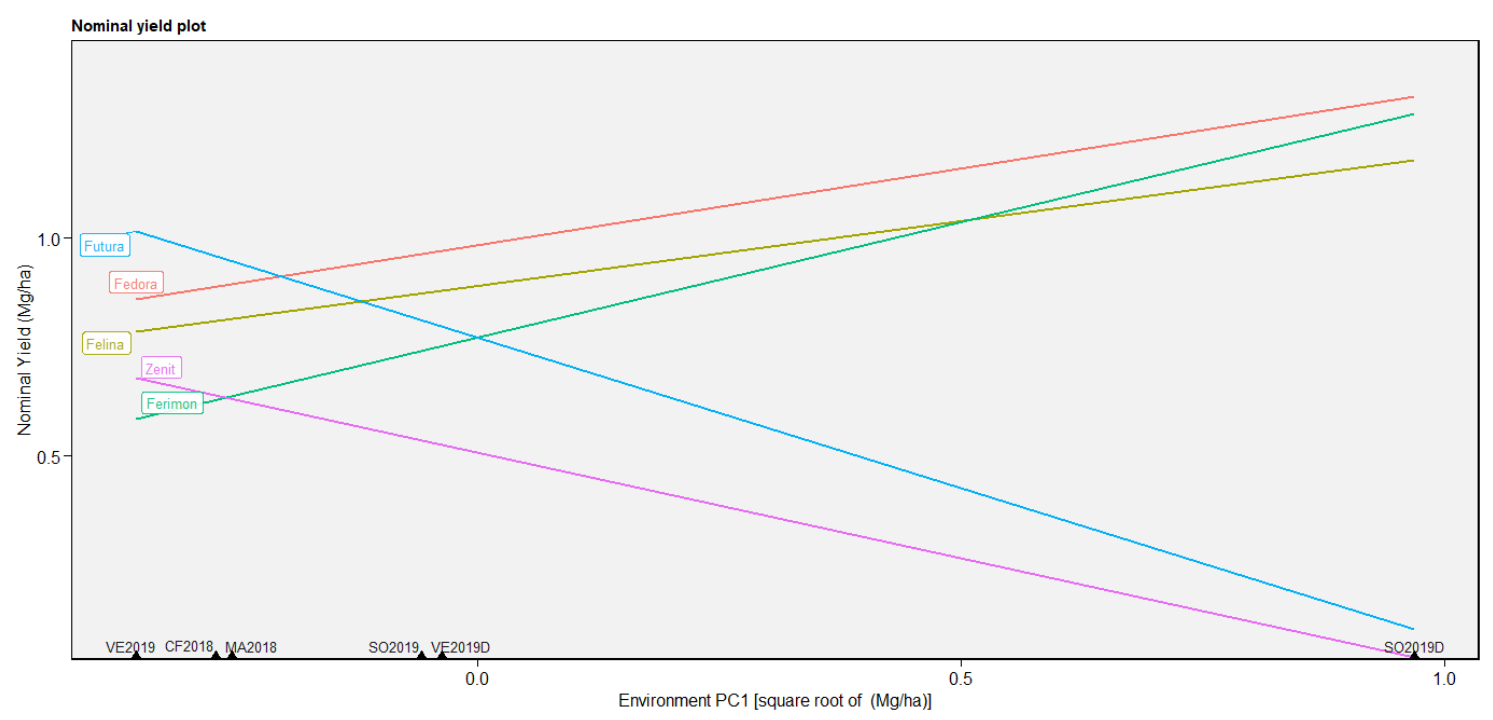

Figure 2. Nominal grain yield for the five hemp cultivars as a function of the environment scores of the first interaction principal component axis (IPCA1).

GEI influence on seed yield was clearly shown using a WAAS biplot (Figure 3), representing the four classes of genotype/environments for a joint interpretation of performance and stability for seed yield. Among the cultivars, Zenit was the only one in the first quadrant, where the most unstable genotypes (that contribute much to GEI) and environments with high discrimination ability should be included, both with a potential seed yield below the grand mean.

Concerning the other cultivars, Futura and Ferimon, located on the border between the first and second quadrants, showed a seed yield very close to the grand mean but with very high instability, evidenced by the high WAAS values. No cultivars were in the second or third quadrant, where genotypes with high seed yield potential but an elevated degree of instability, and with low yield potential and wide adaptability across environments should be included, respectively, due to their low values of WAAS. Felina and Fedora, in the fourth quadrant, showed an above-mean seed yield, and Felina, in particular, showed the lowest value of WAAS among the cultivars, evidencing the best adaptation suitability. Among the environments, SO2019D deserves particular attention since, in addition to providing a significantly higher seed yield potential than the grand mean, it presented a good discrimination ability of the cultivars. CF2018, included in the third quadrant and close to the left corner, can be considered the least productive environment for seed yield, with a very low discrimination ability of the cultivars. The behaviour of locations VE2019 and SO2019 is very interesting, which, in correspondence to the delayed sowing time (VE2019D in quadrant IV and SO2019D in quadrant II), significantly improved their seed yield (Figure 3). 


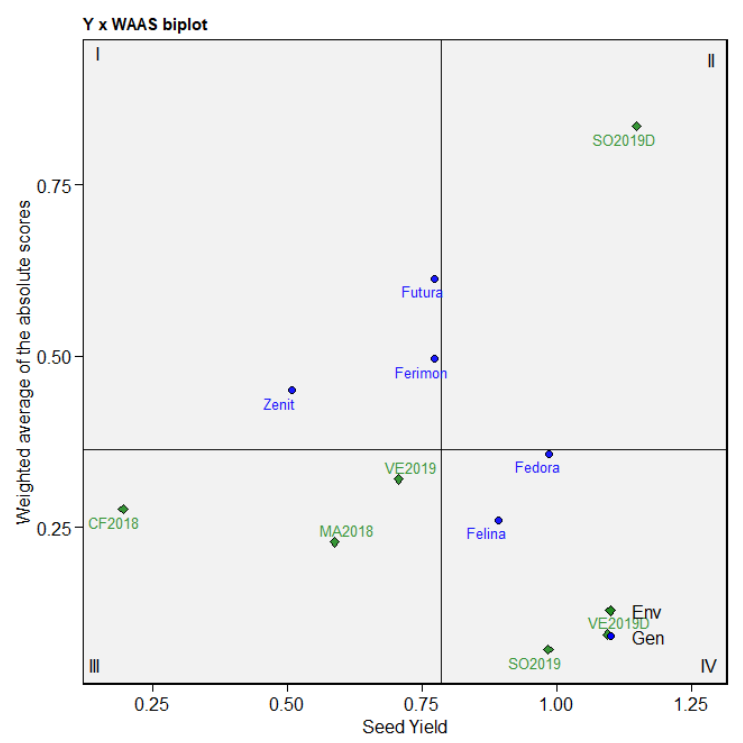

Figure 3. Biplot of the seed yield $\left(\mathrm{Mg} \mathrm{ha}^{-1}\right)$ vs. the WAAS of 5 cultivars evaluated in 6 environments.

Aboveground total plant biomass appeared to be less influenced by environmental factors than the seed yield, with $60 \%$ of character variance due to environmental effects. The genotype and GEI were responsible for a similar amount of variance, at 15 and $20 \%$, respectively (Table 3). Conversely to seed yield, the locations with a conventional sowing time produced the highest plant biomass (Figure 4). In particular, CF2018, SO2019, and MA2018 were included in the second quadrant, with the last showing the highest potential for biomass yield, with an average of $6.9 \mathrm{Mg} \mathrm{ha}^{-1}$. Conversely, VE2019, VE2019D, and SO2019D resulted in the least suitable environments for biomass yield (all below the grand mean), with the last showing the lowest values of the trial $\left(2.1 \mathrm{Mg} \mathrm{ha}^{-1}\right)$. Among genotypes, Futura showed the highest biomass yield $\left(5.0 \mathrm{Mg} \mathrm{ha}^{-1}\right)$, and Zenit the lowest $\left(2.9 \mathrm{Mg} \mathrm{ha}^{-1}\right)$. Felina and Fermion showed the best adaptation suitability and a biomass yield very close to the general average, as did Fedora, which resulted in being the most influenced by the environment for this trait (Figure 4).

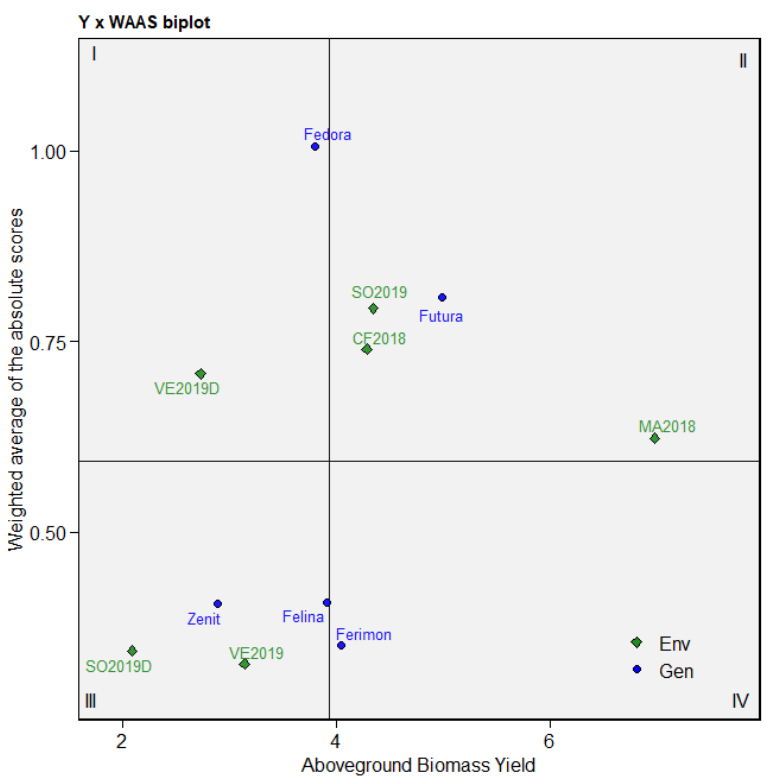

Figure 4. Biplot of the aboveground biomass yield $\left(\mathrm{Mg} \mathrm{ha}^{-1}\right)$ vs. the WAAS of 5 cultivars evaluated in 6 environments. 
Plant height was strongly influenced by both genetic and environmental factors. Genotype accounted for $40 \%$ of the variance in height, and the environment and GEI accounted for 47 and 12\%, respectively (Table 3). At MA2018, with guaranteed water availability at the root level due to the presence of a fairly superficial water table, the plants were taller $(201 \mathrm{~cm}$, on average). The mean plant height by cultivar ranged from 140 to $183 \mathrm{~cm}$, with Futura, the latest cultivar, exhibiting taller plants (Table 2).

The number of days from emergence to flowering had a very strong genetic component, with $61 \%$ of variation, confirming that this character was largely determined by genotype, whereas the environment and GEI components accounted for 27 and $12 \%$ of variation, respectively (Table 3). There were about 61 days, on average, from emergence to flowering; among the earliest was Fedora (53 days), and the latest, Futura (75 days). The latter was confirmed as the latest among the cultivars tested, with the longest period registered at SO2019 (94 days). Regarding the environments, VE2019D showed the average shortest period (44 days), and MA2018 the longest (72 days) (Table 2).

The environment component showed $59 \%$ of variation in the duration of the period from emergence to maturity, $30 \%$ for GEI, and only $11 \%$ for the genotype, evidencing that, contrary to emergence to flowering period, the duration of the post-flowering period is mainly influenced by the environment. VE2019D, the environment with a delayed sowing time, showed the shortest period (84 days, on average) and MA2018, due to its specific above-mentioned characteristics, the longest (128 days, on average). Regarding the varieties, Futura recorded the longest average sowing to maturity period (112 days), confirming it to be the latest cultivar in the trial. Fedora, a very early cultivar, showed the shortest period (94 days) (Table 2).

Seed oil content appeared to be substantially influenced by the environment, with $77 \%$ of character variance due to environmental effects. Genotype and GEI were responsible for 7 and $13 \%$ of variance, respectively (Table 3). The seed oil content varied dramatically, from $5.8 \%$ to $35.0 \%$, with a grand mean of $18.9 \%$. Again, the environments with a delayed sowing time showed the highest seed oil content; in particular, at VE2019D the seed reached an average of $28.2 \%$ of oil content. Among the genotypes, the lowest average seed oil content was achieved by Fedora (15.6\%), while the highest, by Futura (25.2\%) (Table 2). Although the seed oil content values obtained in this trial resulted as very variable between environments (Figure S1), the average value is in agreement with the range of values reported in several previous trials [40-43].

The 1000-seed weight varied significantly from 4.1 to $18.9 \mathrm{~g}$, showing about equivalent and highly significant effects of the genotype, environment, and GEI, accounting for 38, 24 , and $25 \%$ of character variance (Table 3). Among the environments, VE2019D had the heaviest seed, with an average of $12.3 \mathrm{~g}$, and MA2018 the lightest (6.2 g). Among the cultivars, Felina showed the lowest average (6.2 g), and Zenit the highest (12.1 g), despite the cultivar rank changing significantly under specific environments due to the importance of GEI for this character (Table 2). The values obtained in this trial (8.9 g per 1000 -seed weight, as the overall mean) are in agreement with the results obtained in recent experiments conducted in different Greek environments with six monoecious varieties (6.2-9 g) [44], as well as in a location of the Rhaetian Alps in Italy [45], and in various Iranian locations [43]. It is worth noting that the seed weight obtained in this trial for each cultivar was significantly lower by about $30-40 \%$ than that of the pre-planting seed (data not shown), which is in agreement with the results obtained by other authors [43,45].

As expected, the variance in specific seed weight showed the largest proportion attributed to environmental effects at $78 \%$. A substantial $9 \%$ of variance is attributed to GEI effects, whereas $3 \%$ is due to genotype (Table 3). The mean specific seed weight varied significantly, from 0.18 to $0.53 \mathrm{~g} \mathrm{dL}^{-1}$, evidencing the wide variability in environmental conditions among the environments during the seed filling period of the cultivars analysed. The environments with a delayed sowing time showed the highest average value, in particular, VE2019D, with $0.41 \mathrm{~g} \mathrm{dL}^{-1}$, whereas CF2018 showed the lowest $\left(0.23 \mathrm{~g} \mathrm{dL}^{-1}\right)$. 
Between genotypes, Futura, with 0.41 , and Felina, with $0.27 \mathrm{~g} \mathrm{dL}^{-1}$, showed the highest and lowest average specific seed weights, respectively (Table 2).

Correlation coefficients and significant relationships are represented as a heat map to visualise strength and directionality among characteristics (Figure 5). Grain yield was strongly statistically significant and positively correlated with two seed characteristics, namely specific weight $\left(r=0.27^{*}\right)$ and oil content $\left(r=0.55^{* * *}\right)$, evidencing the importance of the grain filling period on seed yield and quality. Conversely, seed yield was negatively correlated with aboveground biomass, but to a limited extent $\left(\mathrm{r}=-0.21^{*}\right)$.

The durations of phenological periods, such as days from emergence to full flowering $\left(-0.37^{* * *}\right)$ and full flowering to seed maturity $\left(-0.45^{* * *}\right)$, were significantly and negatively correlated to the seed yield. On the contrary, the full flowering time $\left(0.37^{* * *}\right)$ and sowing time $\left(0.47^{* * *}\right)$, expressed as day of the year (doy), showed a highly positive and statistically significant link with seed yield. A delay in sowing time and flowering showed a positive effect on seed yield, which was negatively affected by the duration of the above phenological periods.

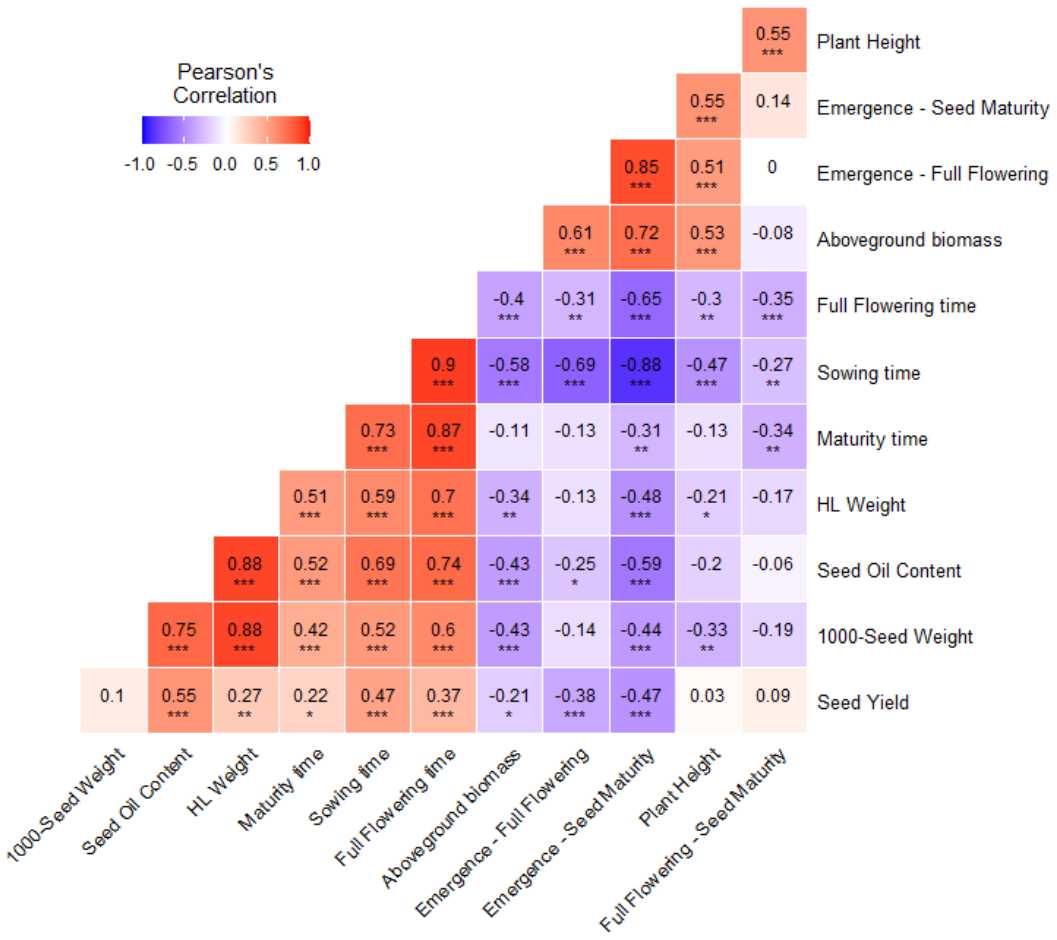

Figure 5. Trait correlations heat maps $\left({ }^{*},{ }^{* *}\right.$ and ${ }^{* * *}$ Significant at the $p<0.05,0.01$ and 0.001 levels, respectively).

Plant biomass was positively correlated with three traits. It was expected that taller plants would accumulate more biomass, and the correlation between biomass and plant height supported this hypothesis $\left(\mathrm{r}=0.53^{* * *}\right)$. As expected, an increase in the duration of the days of emergence to full flowering and emergence to seed maturity periods were positively correlated with aboveground biomass $\left(\mathrm{r}=0.61^{* * *}\right.$ and $0.72^{* * *}$, respectively). Longer phenological periods allowed the plants more time to accumulate a greater quantity of biomass. The sowing time and flowering, expressed as days of the year (doy), showed a significantly negative correlation $\left(-0.58^{* * *}\right.$ and $-0.40^{* * *}$, respectively) with biomass production.

This confirms that the plant has more time to accumulate dry matter with early sowing and flowering, contrary to what was observed for seed yield. Biomass yield also showed negative correlations with the seed characteristics analysed.

Figure 6 reports a bivariate scatterplot of grain yield vs. mean temperature during 150 GDD after the end of flowering, which corresponded, on average, to about the first 
20 days of grain filling for each cultivar. The relationship shows that an increase of mean temperature from 19 to $25^{\circ} \mathrm{C}$ during the first grain filling period led to a progressive decrease in seed yield. Considering this relationship in more detail, it emerges that this trend is valid for the medium-early varieties, but not for Futura, which showed no relationship due to a very limited difference in temperature recorded during the first period of grain filling. These results showed that by delaying the sowing date from April to June, the length of the vegetative phase gradually decreased in all varieties, while the flowering date, especially in Futura, was just slightly delayed (Table S4), which is in agreement with the results obtained by Tang et al. [22] in an environment in central Italy.

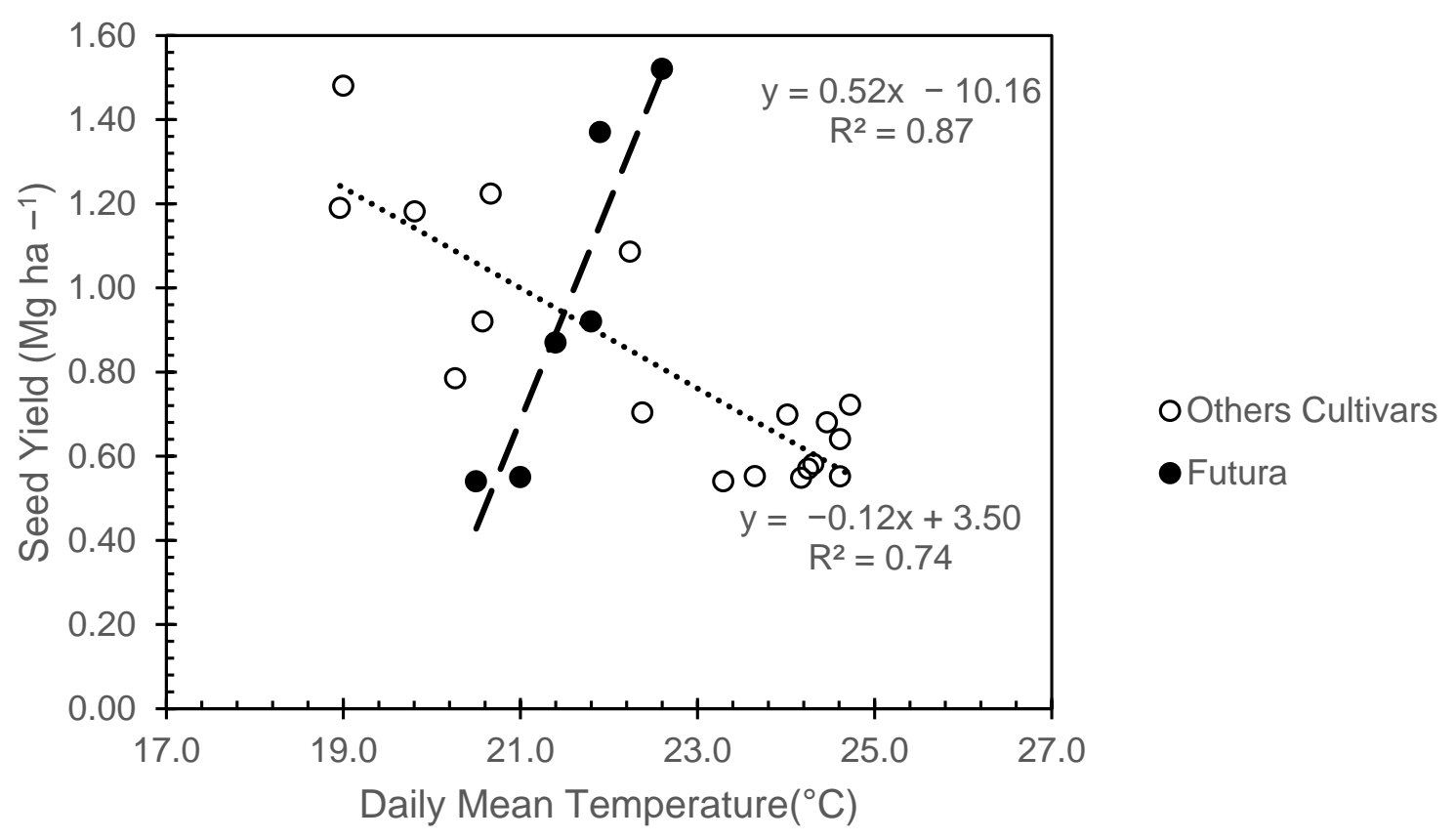

Figure 6. Seed yield as a linear function of the daily mean temperature during 150 GDD after the end of flowering.

\section{Discussion}

The GEI was significant and had a relatively strong influence on the expression of several quantitative traits of agricultural importance in hemp, such as the seed yield, biomass, seed weight, and seed oil content. The GEI could be the basis of yield instability, especially in seed yield, and it may be one of the reasons for inconsistency in seed yield across environments. Therefore, understanding the GEI may identify genotypes that combine high performance and stability across environments (broadly adapted), or in a specific environment, and it is a crucial step for crop spreading. WAASs are important statistical tools for identifying highly productive and stable cultivars (Figures S2-S4). In our study, the WAAS stability index allowed the GEI to be studied and helped to identify the different behaviours of monoecious cultivars across environments. For the seed yield, it allowed two highly productive and broadly adapted cultivars to be identified, namely Fedora and Felina. Furthermore, we identified an environment with good discrimination ability of the cultivars, namely SO2019D. On the other hand, Ferimon was the most stable and broadly adapted cultivar with respect to biomass production. A large number of environments (3 vs. 1) had good discrimination ability of the cultivars' biomass yield stability and adaptability. The WAAS allowed the environments with good discrimination ability to be identified as a function of crop yield (seed and/or biomass), indicating that environments suitable for seed production are different to environments suitable for biomass production. Regardless, more cultivars should be investigated in a large number of environments to achieve a better understanding of the GEI. Gaining a better 
understanding of GEIs for seed production can effectively help guide breeding efforts for developing high-seed cultivars.

The seed yield and seed oil content are undoubtedly the two most important characters for a farmer, with the aim of maximizing the profitability linked to the hemp seed for human consumption, guaranteeing the quantity and quality of the field production (seed) and related products obtained through the first processing (oil and flour). In particular, in the present trial, seed yield and seed oil content are positively correlated, indicating the possibility, through the right crop management technique and a correct cultivar choice, to enhance both.

A delayed sowing, from the end of April to June, showed a positive correlation with seed yield, indicating, in general, an increased grain yield in correspondence to later flowering. This result apparently seems to be in contrast with the results obtained by Tang et al. [22] and Campbell et al. [35] in trials conducted in Italy and Colorado (USA), respectively, where the above relationship resulted as negative. However, in both experiments, characterised mainly by the comparison of different monoecious varieties, the presence of several very late dioecious varieties, which, as is well known, are not suitable for seed yield, has probably conditioned and oriented the above result. Faux et al. [29] showed that with a sowing time progressively postponed from April 16 to the end of June, a progressive reduction of seed yield in monoecious varieties is obtained, in apparent contrast to the present trial. However, that trial was located at over $50^{\circ} \mathrm{N}$ of latitude (Belgium), in contrast to the $46^{\circ} \mathrm{N}$ of our trial. This difference determined a strong difference in many climatic conditions between the two trials, such as average temperatures recorded during the grain filling period. The temperature was very low $\left(13.4^{\circ} \mathrm{C}\right.$ for late sowing) and probably limiting for a satisfactory hemp performance, when compared to the $19.2^{\circ} \mathrm{C}$ recorded during the same period in our trial.

The results provided by Figure 6 and the positive correlation between seed yield and delayed sowing and flowering time, indicate that at least part of the environment and GEI effect on the expression of seed yield in medium-early hemp varieties can be explained by the temperature during the first period of grain filling. In particular, delaying sowing to June would have allowed medium-early varieties to avoid the period with particularly high temperatures $\left(>30^{\circ} \mathrm{C}\right)$ that coincided with the first seed filling period. In fact, this specific phenological phase is considered to be very susceptible to any environmental limitation, which can determine significant negative effects on the quantitative and qualitative seed yield, as already reported for other crops (e.g., [34]). This would also explain the significant increase of seed oil content obtained with a delayed sowing time, confirming that high temperatures after flowering have a detrimental effect on seed oil accumulation, as already observed in hemp [25,46] and as well-known about other crops [47,48], including sunflower, in which seed oil percentage is dramatically reduced by temperatures higher than $>35^{\circ} \mathrm{C}$ due to an increase in the pericarp-to-embryo ratio and a reduction of embryo oil content $[33,49]$.

The heat stress during grain filling in this trial has shown a clear effect on the hemp seed yield for all varieties under study, with the exception of Futura. In this specific case, Futura under delayed sowing had an excessively delayed seed maturation period (Table S4), interacting negatively with other environmental parameters, such as low temperatures, short day lengths, and low light intensities, with a detrimental effect on seed yield.

\section{Conclusions}

The main and general conclusions obtained from this trial are that the WAAS statistical model used provides good indications in relation to the performance and stability of cultivars across environments, in terms of seed yield and total biomass yield, also identifying the environments with the best discrimination ability of the cultivars. A multi-environment trial was conducted on hemp to address some well-known problems, such as low yields and their instability, through identifying the genotypes that combine high performance and stability. 
The importance of the GEI on the expression of seed yield in hemp confirms that the general results and trends obtained, in many cases, have to be reconsidered on the basis of specific varietal and environmental responses. From this trial, it emerged that in the specific environment under study, characterised by a warm, humid climate typical of a northern Mediterranean area, the varieties of Fedora and Felina (medium-early varieties) proved to be the best performing and most stable, and both increased their seed yield with a delayed sowing by about $250{ }^{\circ} \mathrm{C}$ d GDD when compared to the traditional sowing period for the area (about $1400{ }^{\circ} \mathrm{C} \mathrm{d}$ ). The adoption of this strategy, if confirmed by further experiments, could have positive aspects on spreading hemp in the region. Hemp seed, in fact, could be inserted as a new catch crop in the cultivation systems of the area under study, after an autumn-winter crop. Moreover, a delayed sowing will contribute to reducing the plant height, facilitating mechanical harvesting using conventional combines. This solution, however, could simply transfer the heat stress effect from the grain filling to the emergence to seedlings stage; consequently, a study on the sensitivity to high temperature of the seedlings of different cultivars would be necessary. In any case, this solution could be adopted exclusively in locations with sufficient water availability or irrigation facilities in correspondence to seed emergence.

If the aim of hemp cultivation is a dual-purpose crop, the current cultivar availability does not provide a fully satisfactory solution for the environment under study. A good compromise could be a late monoecious cultivar (such as Futura in the present trial), which, if sown early enough, could certainly provide notable results for biomass production and acceptable results for seed production. However, in this case, the plants could easily exceed two meters in height, exacerbating the problems of mechanical seed harvesting.

Supplementary Materials: The following are available online at https:/ / www.mdpi.com/article/ 10.3390/agronomy11071424/s1, Figure S1: WAAS biplot for Seed oil content. Futura showed the highest seed oil content and it depends on environments. Among environments, CF2018 showed the lowest seed oil content, whereas delaying in sowing time increased seed oil content (highest values in SO2019D and VE2019D), Figure S2: AMM1 biplot 1 (left) and 2 (right) for Seed Yield, Figure S3: GGE biplot type 2 Mean performance vs. stability for seed yield, Figure S4: Cross-validation for the full AMMI-family model (1000 re-samples were performed for each cross-validation). AMMI3 model was the best model for seed yield prediction, Table S1: Main soil characteristics of the experimental locations, Table S2: Monthly mean temperature and total rainfall during the hemp crop cycle for each environment of the previous 28-year period (1992-2019), Table S3: Main cropping management techniques adopted for hemp cultivation, Table S4: Main phenological parameters in multiyear trial.

Author Contributions: Conceptualization, M.B.; methodology, M.B. and C.F.; software, C.F.; validation, C.F.; formal analysis, C.F.; investigation, M.B. and F.Z.; data curation, F.Z., G.D. and B.P.; writing-original draft preparation, M.B. and C.F.; writing—review and editing, M.B., C.F. and F.D.; supervision, F.D. and C.C.; project administration, M.B. and C.C.; funding acquisition, M.B. All authors have read and agreed to the published version of the manuscript.

Funding: This research was funded by Agenzia Regionale per lo Sviluppo Rurale del Friuli Venezia Giulia (ERSA—Friuli Venezia Giulia, Regional Agricultural Services, Gorizia, Italy), grant number CUP G24I19000620002.

Data Availability Statement: The data presented in this study are contained within the article or Supplementary Materials. Raw data are available on request from the corresponding author.

Acknowledgments: The authors would like to thank Andrea Fabris for the laboratory analysis of seeds.

Conflicts of Interest: The authors declare no conflict of interest.

\section{References}

1. Salentijn, E.M.J.; Zhang, Q.; Amaducci, S.; Yang, M.; Trindade, L.M. New Developments in Fiber Hemp (Cannabis sativa L.) Breeding. Ind. Crop. Prod. 2015, 68, 32-41. [CrossRef]

2. Schluttenhofer, C.; Yuan, L. Challenges towards Revitalizing Hemp: A Multifaceted Crop. Trends Plant Sci. 2017, 22, 917-929. [CrossRef] [PubMed] 
3. Hu, H.; Liu, H.; Liu, F. Seed Germination of Hemp (Cannabis sativa L.) Cultivars Responds Differently to the Stress of Salt Type and Concentration. Ind. Crop. Prod. 2018, 123, 254-261. [CrossRef]

4. Hu, H.; Liu, H.; Du, G.; Fei, Y.; Deng, G.; Yang, Y.; Feihu, L. Fiber and Seed Type of Hemp (Cannabis sativa L.) Responded Differently to Salt-Alkali Stress in Seedling Growth and Physiological Indices. Ind. Crop. Prod. 2019, 129, 624-630. [CrossRef]

5. Meers, E.; Ruttens, A.; Hopgood, M.; Lesage, E.; Tack, F.M.G. Potential of Brassic rapa, Cannabis sativa, Helianthus annuus and Zea mays for Phytoextraction of Heavy Metals from Calcareous Dredged Sediment Derived Soils. Chemosphere 2005, 61, 561-572. [CrossRef]

6. Rheay, H.T.; Omondi, E.C.; Brewer, C.E. Potential of Hemp (Cannabis sativa L.) for Paired Phytoremediation and Bioenergy Production. GCB Bioenergy 2020, 13, 525-536. [CrossRef]

7. Soudek, P.; Valenova, S.; Vanek, T. Study of radiophytoremediation on heavily polluted area in South Bohemia. In Uranium in the Environment; Springer: Berlin/Heidelberg, Germany, 2006; pp. 519-524.

8. Campbell, S.; Paquin, D.; Awaya, J.D.; Li, Q.X. Remediation of Benzo[a]Pyrene and Chrysene-Contaminated Soil with Industrial Hemp (Cannabis sativa). Int. J. Phytoremediation 2002, 4, 157-168. [CrossRef] [PubMed]

9. Mirizzi, F.; Troyano, V. Hemp Cultivation E Production in Europe in 2018; European Industrial Hemp Association (EIHA): Brussels, Belgium, 2018.

10. Official Journal of the European Union. REGULATION (EU) No 1307/2013 OF THE EUROPEAN PARLIAMENT AND OF THE COUNCILof 17 december 2013 establishing rules for direct payments to farmers under support schemes within the framework of the common agricultural policy and repealing Council Regulation (EC) No 637/2008 and Council Regulation (EC) No 73/2009. L 347/608-L 347/670: 20.12.2013. Available online: https:/ / eur-lex.europa.eu/legal-content/EN/TXT/?uri=celex:32013R1307 (accessed on 30 May 2021).

11. European Commission EU Plant Variety Database. Available online: https://ec.europa.eu/food/plant/plant_propagation_ material/plant_variety_catalogues_databases/search/public/index.cfm (accessed on 30 May 2021).

12. Ministero Della Salute. Circolare 15314-P Del 22/05/2009. In Produzione e Commercializzazione Di Prodotti a Base Di Semi Di Canapa per l'utilizzo Nei Settori Dell'alimentazione Umana; Ministero della Salute [Italian Ministry of Health]: Rome, Italy, 2009.

13. Ministero Della Salute. Decreto 4 novembre 2019. Definizione di livelli massimi di tetraidrocannabinolo (THC) negli alimenti. In 15-1-2020 Gazzetta Ufficiale Della Repubblica Italiana Serie Generale—n. 11; Istituto Poligrafico E Zecca Dello Stato: Rome, Italy, 2020.

14. Grassi, G. Varietà Di Canapa, Stato Attuale e Prospettive [Hemp Varieties, Actual Situation and Perspectives]. Seme Ric. Tecnol. E Destin. Produzioni 2017, 10, 30-41. (In Italian)

15. Struik, P.C.; Amaducci, S.; Bullard, M.J.; Stutterheim, N.C.; Venturi, G.; Cromack, H.T.H. Agronomy of Fibre Hemp (Cannabis sativa L.) in Europe. Ind. Crop. Prod. 2000, 11, 107-118. [CrossRef]

16. Cappelletto, P.; Brizzi, M.; Mongardini, F.; Barberi, B.; Sannibale, M.; Nenci, G.; Poli, M.; Corsi, G.; Grassi, G.; Pasini, P. Italy-Grown Hemp: Yield, Composition and Cannabinoid Content. Ind. Crop. Prod. 2001, 13, 101-113. [CrossRef]

17. Grassi, G. La Canapa Monoica a Confronto Con Varietà Dioiche. L'Informatore Agrar. 2004, 20, 57-61.

18. Allavena, D. Fibranova, Nuova Varieta Di Canapa Ad Alto Contenuto Di Fibra. Sementi Elette 1961, 5, 34-44.

19. Amaducci, S.; Errani, M.; Venturi, G. Confronto Tra Genotipi Monoici e Dioici Di Canapa. L'Informatore Agrar. 1998, $26,39-42$.

20. Del Gatto, A.; Laureti, D.; Crescentini, P. Un Biennio Di Valutazione Di Varietà Di Canapa. L'Informatore Agrar. $2001,16,39-42$.

21. Aubin, M.-P.; Seguin, P.; Vanasse, A.; Lalonde, O.; Tremblay, G.F.; Mustafa, A.F.; Charron, J.-B. Evaluation of Eleven Industrial Hemp Cultivars Grown in Eastern Canada. Agron. J. 2016, 108, 1972-1980. [CrossRef]

22. Tang, K.; Struik, P.C.; Yin, X.; Thouminot, C.; Bjelková, M.; Stramkale, V.; Amaducci, S. Comparing Hemp (Cannabis sativa L.) Cultivars for Dual-Purpose Production under Contrasting Environments. Ind. Crop. Prod. 2016, 87, 33-44. [CrossRef]

23. Tang, K.; Struik, P.C.; Yin, X.; Calzolari, D.; Musio, S.; Thouminot, C.; Bjelková, M.; Stramkale, V.; Magagnini, G.; Amaducci, S. A Comprehensive Study of Planting Density and Nitrogen Fertilization Effect on Dual-Purpose Hemp (Cannabis sativa L.) Cultivation. Ind. Crop. Prod. 2017, 107, 427-438. [CrossRef]

24. Baldini, M.; Ferfuia, C.; Piani, B.; Sepulcri, A.; Dorigo, G.; Zuliani, F.; Danuso, F.; Cattivello, C. The Performance and Potentiality of Monoecious Hemp (Cannabis sativa L.) Cultivars as a Multipurpose Crop. Agronomy 2018, 8, 162. [CrossRef]

25. Baldini, M.; Ferfuia, C.; Zuliani, F.; Danuso, F. Suitability Assessment of Different Hemp (Cannabis sativa L.) Varieties to the Cultivation Environment. Ind. Crop. Prod. 2020, 143, 111860. [CrossRef]

26. Finlay, K.W.; Wilkinson, G.N. The Analysis of Adaptation in a Plant-Breeding Programme. Aust. J. Agric. Res. 1963, 14, 742. [CrossRef]

27. Zobel, R.W.; Wright, M.J.; Gauch, H.G. Statistical Analysis of a Yield Trial. Agron. J. 1988, 80, 388-393. [CrossRef]

28. Olivoto, T.; Lúcio, A.D.C.; Silva, J.A.G.; Marchioro, V.S.; Souza, V.Q.; Jost, E. Mean Performance and Stability in Multi-Environment Trials I: Combining Features of AMMI and BLUP Techniques. Agron. J. 2019, 111, 2949-2960. [CrossRef]

29. Faux, A.-M.; Draye, X.; Lambert, R.; d'Andrimont, R.; Raulier, P.; Bertin, P. The Relationship of Stem and Seed Yields to Flowering Phenology and Sex Expression in Monoecious Hemp (Cannabis sativa L.). Eur. J. Agron. 2013, 47, 11-22. [CrossRef]

30. Gunawardena, T.A.; Fukai, S.; Blamey, F.P.C. Low Temperature Induced Spikelet Sterility in Rice. I. Nitrogen Fertilisation and Sensitive Reproductive Period. Aust. J. Agric. Res. 2003, 54, 937. [CrossRef]

31. Lafitte, H.R.; Edmeades, G.O. Temperature Effects on Radiation Use and Biomass Partitioning in Diverse Tropical Maize Cultivars. Field Crop. Res. 1997, 49, 231-247. [CrossRef] 
32. Ohnishi, S.; Miyoshi, T.; Shirai, S. Low Temperature Stress at Different Flower Developmental Stages Affects Pollen Development, Pollination, and Pod Set in Soybean. Environ. Exp. Bot. 2010, 69, 56-62. [CrossRef]

33. Rondanini, D.; Savin, R.; Hall, A.J. Dynamics of Fruit Growth and Oil Quality of Sunflower (Helianthus annuus L.) Exposed to Brief Intervals of High Temperature during Grain Filling. Field Crop. Res. 2003, 83, 79-90. [CrossRef]

34. Rondanini, D.; Mantese, A.; Savin, R.; Hall, A.J. Responses of Sunflower Yield and Grain Quality to Alternating Day/Night High Temperature Regimes during Grain Filling: Effects of Timing, Duration and Intensity of Exposure to Stress. Field Crop. Res. 2006, 96, 48-62. [CrossRef]

35. Campbell, B.J.; Berrada, A.F.; Hudalla, C.; Amaducci, S.; McKay, J.K. Genotype $\times$ Environment Interactions of Industrial Hemp Cultivars Highlight Diverse Responses to Environmental Factors. Agrosyst. Geosci. Environ. 2019, 2, 1-11. [CrossRef]

36. Mediavilla, V.; Jonquera, M.; Schmid-Slembrouck, I.; Soldati, A. Decimal Code for Growth Stages of Hemp(Cannabis sativa L.). J. Int. Hemp Assoc. 1998, 5, 68-74.

37. R Core Team. R: A Language and Environment for Statistical Computing; R Foundation for Statistical Computing: Vienna, Austria, 2020.

38. Olivoto, T.; Lúcio, A.D. Metan: An R Package for Multi-Environment Trial Analysis. Methods Ecol. Evol. 2020, 11, 783-789. [CrossRef]

39. Malosetti, M.; Ribaut, J.-M.; van Eeuwijk, F.A. The Statistical Analysis of Multi-Environment Data: Modeling Genotype-byEnvironment Interaction and Its Genetic Basis. Front. Physiol. 2013, 4, 44. [CrossRef] [PubMed]

40. Kriese, U.; Schumann, E.; Weber, W.E.; Beyer, M.; Brühl, L.; Matthäus, B. Oil Content, Tocopherol Composition and Fatty Acid Patterns of the Seeds of 51 Cannabis sativa L. Genotypes. Euphytica 2004, 137, 339-351. [CrossRef]

41. Galasso, I.; Russo, R.; Mapelli, S.; Ponzoni, E.; Brambilla, I.M.; Battelli, G.; Reggiani, R. Variability in Seed Traits in a Collection of Cannabis sativa L. Genotypes. Front. Plant Sci. 2016, 7, 688. [CrossRef]

42. Vonapartis, E.; Aubin, M.-P.; Seguin, P.; Mustafa, A.F.; Charron, J.-B. Seed Composition of Ten Industrial Hemp Cultivars Approved for Production in Canada. J. Food Compos. Anal. 2015, 39, 8-12. [CrossRef]

43. Abdollahi, M.; Sefidkon, F.; Calagari, M.; Mousavi, A.; Mahomoodally, M.F. A Comparative Study of Seed Yield and Oil Composition of Four Cultivars of Hemp (Cannabis sativa L.) Grown from Three Regions in Northern Iran. Ind. Crop. Prod. 2020, 152, 112397. [CrossRef]

44. Tsaliki, E.; Kalivas, A.; Jankauskiene, Z.; Irakli, M.; Cook, C.; Grigoriadis, I.; Panoras, I.; Vasilakoglou, I.; Dhima, K. Fibre and Seed Productivity of Industrial Hemp (Cannabis sativa L.) Varieties under Mediterranean Conditions. Agronomy 2021, $11,171$. [CrossRef]

45. Pavlovic, R.; Panseri, S.; Giupponi, L.; Leoni, V.; Citti, C.; Cattaneo, C.; Cavaletto, M.; Giorgi, A. Phytochemical and Ecological Analysis of Two Varieties of Hemp (Cannabis sativa L.) Grown in a Mountain Environment of Italian Alps. Front. Plant Sci. 2019, 10. [CrossRef]

46. Anwar, F.; Latif, S.; Ashraf, M. Analytical Characterization of Hemp (Cannabis sativa) Seed Oil from Different Agro-Ecological Zones of Pakistan. J. Am. Oil Chem. Soc. 2006, 83, 323-329. [CrossRef]

47. Connor, D.J.; Hall, A.J. Sunflower Physiology. In Sunflower Technology and Production; Schneiter, A.A., Ed.; American Society of Agronomy, Crop Science Society of America, Soil Science Society of America: Madison, WI, USA, 1997; Volume 35, pp. 113-182.

48. Harris, H.C.; McWilliam, J.R.; Mason, W.K. Influence of Temperature on Oil Content and Composition of Sunflower Seed. Aust. J. Agric. Res. 1978, 29, 1203. [CrossRef]

49. Angeloni, P.; Aguirrezábal, L.; Echarte, M.M. Assessing the Mechanisms Underlying Sunflower Grain Weight and Oil Content Responses to Temperature during Grain Filling. Field Crop. Res. 2021, 262, 108040. [CrossRef] 